\title{
Finding a minimal transitive reduction in a strongly connected digraph within linear time
}

\section{Report}

Author(s):

Simon, Klaus

Publication date:

1989

Permanent link:

https://doi.org/10.3929/ethz-a-000628392

Rights / license:

In Copyright - Non-Commercial Use Permitted

Originally published in:

ETH, Eidgenössische Technische Hochschule Zürich, Departement Informatik, Institut für Theoretische Informatik 103 


\section{ETH}

Eidgenössische

Technische Hochschule

Zürich
Departement Informatik Institut für

Theoretische Informatik

Klaus Simon Finding

a Minimal Transitive Reduction in a Strongly Connected Digraph within Linear Time

April 1989

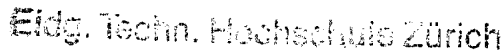

Infometrothother

ETr-zontuth

Con

103 
Author's adress:

Institut für Theoretische Informatik ETH-Zentrum

$\mathrm{CH}-8092$ Zürich, Switzerland

(C) 1989 Departement Informatik, ETH Zürich 


\begin{abstract}
This paper describes an algorithm for finding a minimal transitive reduction $G_{\text {red }}$ of a given directed graph $G$, where $G_{r e d}$ means a subgraph of $G$ with the same transitive closure as $G$ but itself not contains a proper subgraph $G_{1}$ with the same property too. The algorithm uses depth-first search and two graph transformations preserving the transitive closure to achieve a time bound of $O(n+m)$, where $n$ stands for the number of vertices and $m$ is the number of the edges.
\end{abstract}

\title{
1. Introduction
}

One of the classical topic in computer science is computing the path information of a directed graph (digraph) $G=G(V, E)$, where $V(E)$ represents the vertex (edge) set. In this connection there are two extreme points of view:

i) A minimum query time representation. Determine the unique digraph $G^{*}\left(V, E^{*}\right)$ which contains a directed edge from $v$ to $w$ if and only if there is a directed path from $v$ to $w$ in $G$.

ii) A minimum storage representation. Determine the "edge minimal" graph $G_{r}\left(V, E_{r}\right)$ with the property $G_{r}^{*}=G^{*}$.

The graph $G^{*}$ is traditionally called transitive closure of $G$. The appellation for $G_{r}$ is not unique. In former papers (MOYES AND THOMPSON, HsU) the indication minimum corresponding graph is used for $G_{r}$ but nowadays $G_{r}$ is normally named transitive reduction of $G$. In order to avoid baffling in the application of the conception transitive reduction we have first of all to differentiate between the adjectives "minimal" and "minimum" related to $G_{r}$ or $E_{r}$ and on the other hand between $E_{r} \subseteq E$ and $E_{r} \subseteq V \times V$. In terms of a set "minimal" means a set property, whereas "minimum" refers to the size of the set. Especially we say $G_{r}$ is a minimal transitive reduction of $G$ if and only if $G_{r}^{*}=G^{*}$ and there is no proper subgraph $G_{1}$ of $G_{r}$ with $G_{1}^{*}=G_{r}^{*}$. In other words a minimal transitive reduction is a subgraph $G_{r}$ of $G$ with $G_{r}^{*}=G^{*}$ and no reducible 
edge $(v, w)$ in it, where $(v, w)$ is reducible in $G_{r}$ if and only if there is a simple path $P=v_{0}, \ldots, v_{s}$ from $v=v_{1}$ to $w=v_{s}$ in $G_{r}-(v, w)$. A minimum transitive reduction is a transitive reduction of minimum size.

The second point to be regarded is the condition $E_{r} \subseteq E$ or $E_{r} \subseteq$ $V \times V$. AHo, GAREY AND Ullman have shown, that this makes no difference on acyclic digraphs. Moreover, on acyclic digraphs a minimal transitive reduction is also a minimum transitive reduction. But in a strongly connected digraph - a digraph $G(V, E)$ with $E^{*}=V \times V$ the problem of finding a transitive reduction becomes trivial demanding on condition that $E_{r} \subseteq V \times V$ since every permutation of the nodes is a minimal and a minimum solution. Otherwise, on condition $E_{r} \subseteq E$ the deciding whether digraph $G$ has a minimum transitive reduction with at most $c$ edges - for a given $c-$ is NP-complete. Note for example: A hamiltonian circuit is always a minimum transitive reduction. This is a result by SAHNI. But back again to our problem. We are describing an linear algorithm for finding a minimal transitive reduction $G_{r}\left(V, E_{r}\right)$ of a given strongly connected digraph $G(V, E)$, where the transitive reduction is definded on condition $E_{r} \subseteq E$ and "minimal" stands for containing no reducible edge. The simple idea which the algorithm follows is to delete is many edges as possible without changing the transitive closure. The lgorithm is based on an iterative use of depth-first search, TARJAN, 972 , and two kinds of graph transformations which do not change the ransitive reduction.

The paper is divided into several sections. Section 2 contains some notations and a short description of depth-first search on directed graphs. In section 3 we develop an algorithm to delete all reducible edges which are not in the tree constructed by depth-first search. Section 4 gives a method to find reducible tree arcs by a special version of depth-first search. Section 5 contains a short conclusion and open problems.

\section{Notations and Basic Concepts.}

As usual we need some notations. First of all the graph-theoretic definitions which are more or less standard. For an advanced reader conversant with digraphs and depth-first search it is not necessary to read the section. A (directed) graph $G=G(V, E)$ is an ordered couple consisting of a set of vertices (nodes) $V, V=\{1, \ldots, n\}$, and a set of edges (arcs) $E$, $E \subseteq V \times V$. A graph is always a simple graph which contains no parallel edges or self-loops - $\operatorname{arcs}$ of the form $(v, v)$. We say edge $(v, w)$ leaves $v$, 
enters $w$ and joins $v$ with $w$. Unless we specify otherwise, any subgraph is the subgraph induced by its vertex set. A path $P$ in $G$ from vertex $v_{0}$ to vertex $v_{s}$ is a sequence of vertices $v_{0}, v_{1}, \ldots, v_{s}$ such a way that $\left(v_{i-1}, v_{i}\right)$ is an edge for $i \in\{1,2, \ldots, s\} ; s$ is the length of the path. The path $P$ is simple if all its vertices are pairwise distinct. A path $v_{0}, \ldots, v_{s}$ is a cycle if $s>1$ and $v_{0}=v_{s}$ and a simple cycle if in addition $v_{1}, \ldots, v_{s-1}$ are pairwise distinct. A graph without cycles is acyclic. Let be $A \subseteq E$ then we write $v \stackrel{*}{\rightarrow} w$ if there is a path from $v$ to $w$ using only $\operatorname{arcs}$ of $A$. If there is $v \stackrel{*}{\boldsymbol{E}} w$ ( or $v \stackrel{*}{\rightarrow} w$ or simply $v \stackrel{*}{\longrightarrow} w$ ) then $w$ is said reachable from $v$. If $v \stackrel{*}{\longrightarrow} w$ is a simple path with length greater than 0 then we write $v \stackrel{+}{\longrightarrow} w$. A single edge $(v, w)$ is also written as $v \rightarrow w$. A digraph $G$ is strongly connected if it satisfies the conditions $v \stackrel{*}{\longrightarrow} w \stackrel{*}{\longrightarrow} v$ for all $v, w \in V$.

A (directed, rooted) tree $T$ is an acyclic digraph with one distinguished vertex $r$ called the root $r$ such a way that $r \stackrel{+}{\longrightarrow} v$ for all vertices $v, v \neq r$, and no edges enter $r$. Please note: In a tree exactly one arc enters every other vertex as the root. A tree vertex with no existing edges is a leaf. Let $(v, w)$ be a tree edge, then $v$ is the father of $w$ and $w$ is a son of $v$. If $v \underset{T}{\stackrel{*}{\longrightarrow}} w$, then $v$ is an ancestor of $w$ and $w$ is a descentant of $v$. A tree node $z$ is called a common ancestor of the node $v$ and $w$ if and only if $z \stackrel{*}{T} v$ and $z \stackrel{*}{T} w$. The vertex $z$ is the nearest common ancestor of $v$ and $w$ iff there is no other common ancestor $x$ of $v$ and $w$ with $x \neq z$ and $z \underset{T}{\stackrel{*}{\longrightarrow}} x$. A subtree $T_{1}$ of a tree $T$ with root $v$ is the subgraph of $T$ induced by the nodes $\{w \in V \mid v \stackrel{*}{T} w\}$. If a tree $T$ contains all the vertices of $G$, then $T$ is called a spanning tree of $G$. The height of a node $v$ in $T$ is the length of path with maximum length from $v$ to a leaf in the subtree of $v$. A sink tree $S$ is a tree in which the edges are directed from a son to its father or in other words it is

$$
w \stackrel{*}{S} r \quad \forall w \in V
$$

where $\mathbf{r}$ is the root of $S$.

Our algorithm is based on a systematic exploration of a graph. Especially we are using the depth-first search procedure in the version of TARJAN, 1972, to recognize the strongly connected components of a digraph 


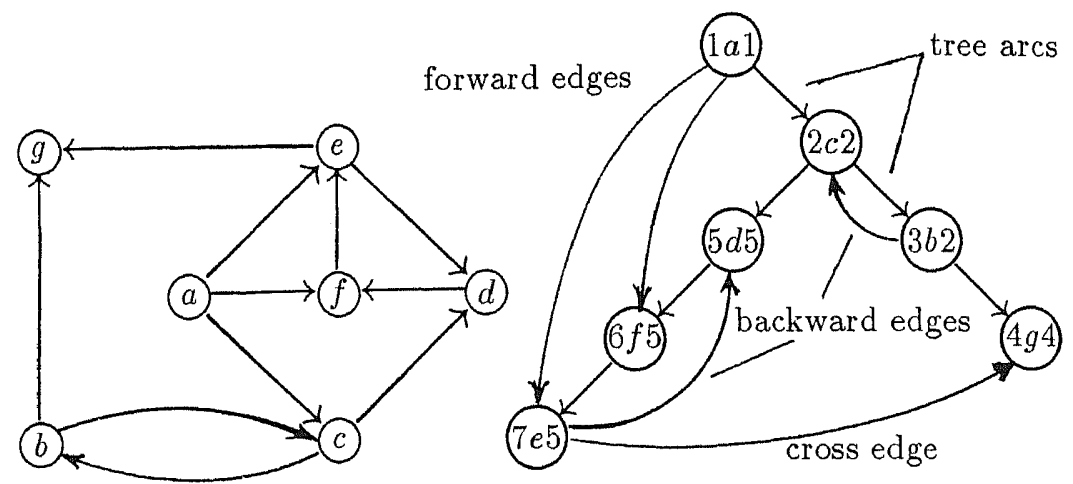

Figure 1

A digraph $G$ and its DFS-tree $T$. The inscription of a vertex $v$ in $T$ is given by $d f$ snum $|v|$ compnum. Throughout this paper the nodes in $T$ are arranged from right to left and from top to bottom relative to their $d f s n u m$. Later the nodes will be identified with their $d f_{s n u m}$.

$G$. Therefore, we need a short description of this version of depth-first search (DFS). It is convenient to formulate DFS as a recursive procedure $D F S(v)$ with vertex $v$ as parameter, see TARJAN, 1972. In general we search for unexplored vertices by traversing an unexplored edge from the most recently reached vertex which still has unexplored edges. The set $R E A C H$ contains the explored vertices. On these conditions DFS has the following main structure

depth-first search

procedure $D F S(v: V)$

begin

add $v$ to $R E A C H$;

for $\forall w$ with $(v, w) \in E$ do

if $w \notin R E A C H$ then $D F S(w) \mathbf{f i}$

od

end

depth-first search

The procedure starts with 


$$
\begin{aligned}
& R E A C H \leftarrow \emptyset ; \\
& D F S(r) ;
\end{aligned}
$$

and marks all vertices reachable from start vertex $r$. But DFS gives some further information about the digraph $G$. In particular, DFS computes a spanning tree DFS-tree with root $r$, a numbering $d f s n u m$ with respect to calling time of procedure DFS and a second numbering compnum with respect to completion time of DFS. With these numberings we partition the edges of the graph into four classes: The tree edges $T$, the forward edges $F$, the backward edges $B$ and the cross edges $C$. This partition is defined by

(i) $\quad(v, w) \in T \Longleftrightarrow w$ unmarked when $(v, w)$ is explored.

(ii) $\quad(v, w) \in F \quad \Longleftrightarrow d f s n u m(v)<d$ fsnum $(w)$

$$
\text { and compnum }(w)<\operatorname{compnum}(v)
$$$$
\text { and }(v, w) \notin T \text {. }
$$

(iii) $\quad(v, w) \in B \quad \Longleftrightarrow d f \operatorname{snum}(w)<d f \operatorname{snum}(v)$

$$
\text { and compnum }(v)<\operatorname{compnum}(w) \text {. }
$$

(iv) $\quad(v, w) \in C \quad \Longleftrightarrow d f \operatorname{snum}(v)>d f \operatorname{snum}(w)$

$$
\text { and compnum }(w)<\operatorname{compnum}(v) \text {. }
$$

In figure 1 we give an example for such a partition. For the proof of this and some further properties of DFS see MEHLHORN, 1984. This is also a good reference for a detailed description of DFS to compute dfsnum, DFS-tree, $B$ and so on, in linear time.

In order to recognize the strongly connected components of a given digraph $G$, the procedure DFS uses a map

$$
\text { lowpoint }: V \rightarrow V \text {. }
$$

defined by

$$
\operatorname{lowpoint}(v)=\min \{d f \operatorname{sn} u m(w) \mid \exists(u, w) \in E \text { with } v \underset{T}{\stackrel{*}{\longrightarrow}} u\} .
$$

Let $v$ be the root of DFS-tree. We then have the following wellknown result: The digraph $G(V, E)$ is strongly connected if and only if

$$
\text { lowpoint }(v)<d f \operatorname{snum}(v) \quad \forall v \in V, v \neq r .
$$


For proof see MEHLHORN, 1984. Let now lowpoint $(v)=w$ then lowpoint $(v)$ is defined by an edge $(u, w) \in E$, where $u$ is a node in the subtree of $v$ with respect to DFS-tree. This edge we indicate as lowedge $(v)$. Now we obtain a special subgraph $G_{\text {low }}\left(V, E_{\text {low }}\right)$ of $G$ by

$$
G_{l o w}=\text { DFS-tree }+\{\text { lowedge }(v) \mid v \in V, v \neq r\} .
$$

For the subgraph $G_{l o w}$ it is easy to see: $G$ is strongly connected if and only if $G_{\text {low }}$ is strongly connected. Note: $G_{\text {low }}$ has at most $2 \cdot n$ edges. Naturally the question arises whether perhaps $G_{l o w}$ is a minimal transitive reduction too? In general this is incorrect, see figure 2.
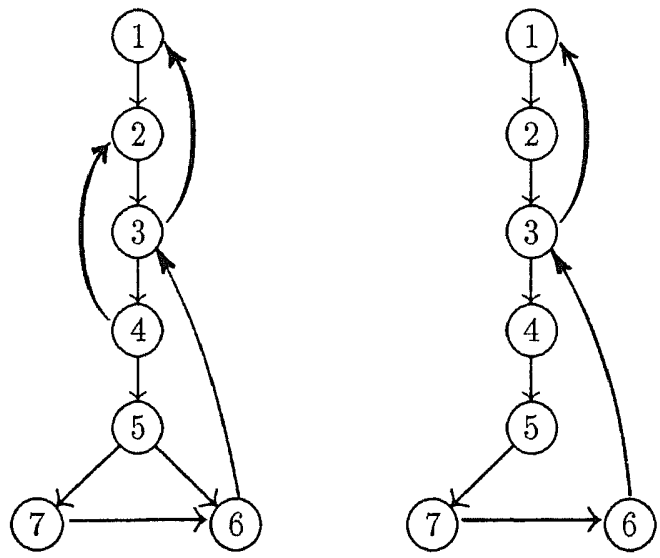

Figure 2: A low-graph and its transitive reduction.

After this preparation we can start with our analysis of finding a minimal transitive reduction.

\section{Deleting Reducible No-tree Arcs}

We begin by exploring $G$, starting DFS at any vertex $v$. Then DFS partitions the edges of $G$ in tree $\operatorname{arcs} T$, forward edges $F$, the backward edges $B$ and cross edges $C$. To simplify the discussion we shall identify vertices using the $d f s n u m$ assigned them by DFS. Now a simple idea for obtaining a minimal transitive reduction is to delete as many reducible edges as possible. For getting a linear time complexity we have to check in time $O(1)$ for each and every selected edge $e$ : Is $e$ reducible? For some 
edges it is not very difficult to answer, i.e. see lemma 1. But for other edges $e$ it is not easy, see lemma 3 . In these cases we give no answer but we replace the edge $e$ by an edge $e^{\prime}$ of a simplier type. The new edge $e^{\prime}$ has the property: $e^{\prime}$ is reducible in $G-e+e^{\prime}$ if and only if $e$ is reducible in $G$. This replacement allows us to postpone the check at a later date. Moreover, if $e^{\prime \prime}$ is not a tree arc then $e^{\prime \prime}$ is reducible in $G$ if and only if $e^{\prime \prime}$ is reducible in $G-e+e^{\prime}$. This implies our general strategy for this chapter. At each step select one edge $e, e \in B \cup C \cup F$, and in time $O(1)$ either test $e$ to be reducible or replace $e$ by another edge $e^{\prime}$. Let's now come to a detailed treatment.

Note: In the following we assume that the DFS-tree is always a subgraph to $G$. In this section we do not delete any tree arcs from $G$. On this condition we get

Lemma 1. Each forward edge $e=(v, w)$ is reducible.

Proof. By definition of forward edges there is a simple path $v \frac{+}{T} w$ with length greater or equal than two. But this is a reducing path for the edge $(v, w)$.

The next lemma is giving us an analogous proposition for backward edges.

Lemma 2. Let $\left\{\left(v, w_{1}\right), \ldots,\left(v, w_{s}\right)\right\}$ be the set of backward edger emanating from node $v$ such a way that $w_{1}<w_{2}<\cdots<w_{s}$ then all the edges $\left(v, w_{2}\right), \ldots,\left(v, w_{s}\right)$ are reducible.

Proof. Remember we have $\operatorname{dfs} u m(v)=v$ for all vertices. Now all

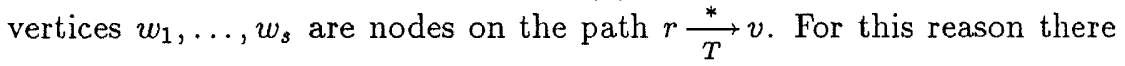
is a reducing path $P=v \rightarrow w_{1} \frac{+}{T} w_{i}$ for each backward edge $\left(v, w_{i}\right)$, $2 \leq i \leq s$.

To check the cross edges we need a more complicated proposition which is given in the following lemma.

Lemma 3. Let $e=(v, z)$ be a cross edge and $w$ is the nearest common ancestor of $v$ and $w$ in the DFS-tree. If $(v, w) \in E$ then $(v, z)$ is reducible in $G$. If $(v, w)$ is not an edge of $G$ we obtain:

1. The edge $(v, z)$ is reducible in $G$ if and only if the edge $e^{\prime}=(v, w)$ is reducible in the digraph $G_{1}=G-(v, z)+(v, w)$. 
2. $G_{1}^{*}=G^{*}$.

3. Let $e^{\prime \prime} \in C \cup B \cup F$ be an edge with $e \neq e^{\prime \prime} \neq e^{\prime}$ then $e^{\prime \prime}$ is reducible in $G$ if and only if $e^{\prime \prime}$ is reducible in $G_{1}$.

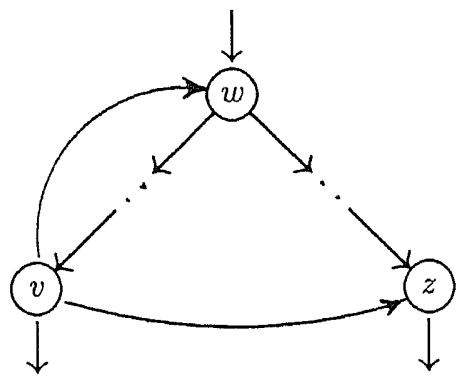

Figure 3

Proof. Let's have a look at figure 3. First of all we see if $(v, w) \in E$ then the reducing path to $(v, z)$ is given by $v \rightarrow w \frac{+}{x} z$.

1. On the other hand let now $(v, w)$ be not an edge of $G$. Further let $G_{2}$ be the graph formed by $G$ by deleting edge $(v, z)$. Assuming $(v, z)$ is reducible in $G$ there is a reducing path $P_{1}$ from $v$ to $z$ in $G$. Since $G$ is strongly connected there is a simple path $P_{2}$ from $z$ to $w$. Joining $P_{1}$ with $P_{2}$ we get a path $P_{3}$ from $v$ to $w$ which does not contain $(v, z)$. But $P_{3}$ is a path in $G_{2}$ and, therefore, there is also a simple path $P_{4}=v \underset{G_{2}}{\longrightarrow} w$ in $G_{2}$. Since $G_{1}=G_{2}+(v, w), P_{4}$ must be a reducing to $(v, w)$ in $G_{1}$. Conversely, suppose $P_{1}$ is a reducing path to $(v, w)$ in $G_{1}$. Extending $P_{1}$ by $w \stackrel{t}{T} z$ we observe a path $P_{2}$ in $G$ from $v$ to $z$. By shrinking cycles we find a reducing path for $(v, z)$ in $G$.

2. In every path $P$ in $G$ which contains the $\operatorname{arc}(v, z)$ we can replace this arc by the path $v \rightarrow w \frac{+}{T} z$ in $G$.

3. Let now $e^{\prime \prime}=(x, y)$ be an edge in $G$, with $e^{\prime \prime} \notin T,(v, w) \neq e^{\prime \prime} \neq(v, z)$. Suppose $e^{\prime \prime}$ is reducible in $G$ and $P_{1}$ is the corresponding reducing path. If $(v, z)$ is not an edge of $P_{1}$ then there is nothing to show. Therefore, we assume $(v, z)$ is an edge of $P_{1}$. Replacing $(v, z)$ in $P_{1}$ by $v \rightarrow w \frac{+}{T} z$ produces a path $P_{2}$ in $G_{1}$ which connects the same endpoints as $P_{1}$. By construction $e^{\prime \prime}$ is not an edge of $P_{2}$. Hence, shrinking cycles gets the 
reducing path to $e^{\prime \prime}$ in $G_{1}$. Conversely, suppose $e^{\prime \prime}=(x, y)$ is reducible in $G_{1}$. Then we consider two cases. The trivial case is given if there is a reducing path $P_{1}$ for the edge $e^{\prime \prime}$ which does not contain the edge $(v, w)$. This path $P_{1}$ exists in $G$ too. In the notrivial case every reducing path $P_{1}$ contains the edge $(v, w)$. Let $u$ be first node after the starting node $w$ on the tree path from $w$ to $v$, thus $w \underset{T}{\longrightarrow} u \stackrel{+}{T} v$. By our presumption about $P_{1}$ we find that the node $x$ cannot be less than $u$. We see this by a short view on the structure of DFS. Let $w=w_{1}, \ldots, w_{s}$, with $w_{1}=r$ and $w_{s}=w$, be the tree path $v \underset{T}{+} w$ from the root $r$ to the vertex $w$. We infer from the definition of depth-first search: If $\left(w_{s-1}, w_{s}\right)$ is a tree edge then it exists no edge $\left(a, w_{s}\right)$ with $a<w_{s}$ and $a \neq w_{i}, 1 \leq i \leq s-1$. In other words, the vertices $w_{1}, \ldots, w_{s}$ builds a vertex separator for all nodes $a$ and $b$ with $a<w_{s} \leq b$. That means every path connecting $a$ and $b$ passes through at least one vertex $w_{i}, 1 \leq i \leq s-1$. Now we can show our proposition. If we assume $x<u$ conversely to our proposition then we observe with $a=x$ and $b=v$ : The reducing path $P_{1}$ from $x$ over $v$ and $w$ to $y$ must pass through a vertex $w_{i}, 1 \leq i \leq s-1$, before reaching $v$. But the part $w_{1} \stackrel{+}{\longrightarrow} v \rightarrow w$ in the path $P_{1}$ can be replaced by $w_{i} \stackrel{+}{T} w$ to get a reducing path which does not contain $(v, w)$ in contradiction to our presumption that no such path exists. Therefore, it must hold $x \geq u$. The same argument as above shows further that there is a path $W$ in $G$ from $z$ to $w$ which does not contain a node $h$ with $h>u$. Since the edge $e^{\prime \prime}=(x, y)$ cannot be an edge of this path $W$. Consequently we can replace the $\operatorname{arc}(v, w)$ in path $P_{1}$ by $(v, z)$ and $W$ to get a path $P_{2}$ from $x$ to $y$ in $G$. Shrinking cycles in $P_{2}$ gives us the reducing path $P_{3}$ for the edge $(x, y)$ in $G$ and the lemma follows.

Now lemma 3 allows us the following strategy to find and delete reducible edge. First delete all forward edges by lemma 1. Then delete or replace all cross edges by lemma 3 . We use a map standfor to store up the origin $(v, z)$ of a new edge $(v, w)$. Now the graph contains only backward edges others than tree arcs. In the third step we delete all backward edges except one for each node $v$ by lemma 1 . In the following we call this resulting digraph $G^{\prime}$. Note that the DFS-tree for $G$ is also a DFStree for $G^{\prime}$ and $G^{\prime}$ is strongly connected by induction on the construction process.

Remark: Figure 4 shows an easy example that Lemma 3.3 is not correct for tree arcs. Therefore, we need a separate method to find reducible tree 

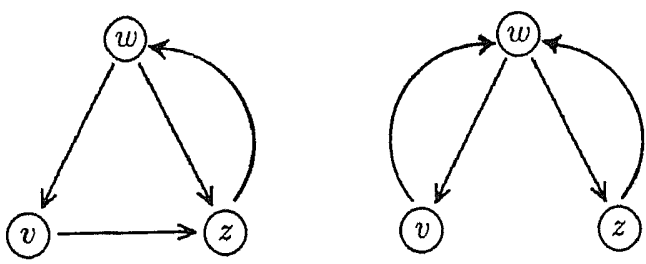

Figure 4: $(v, z)$ is reducible in $G$ but not in $G_{1}$.

arcs, see chapter 4 .

The diagraph $G^{\prime}$ contains the DFS-tree and at most one backward edge $(v, w)$ for every node $v$. Now we examine this edge $e=(v, w)$ to its reducibility. We shall give a bottom up procedure to make this. Therefore, we start in a leaf $v$ and this case is easy, because it is clear that the only edge emanating $v$ cannot be reducible. The next lemma describes the situation in which $v$ is an inner node.

Lemma 4. Let $(v, w)$ be a backward edge in $G, x$ be a descendant of $v$ with $v \neq x$ and $z$ be a vertex with $v \stackrel{+}{T} x \rightarrow z$ and $(x, z) \in E$. (In other words, $(x, z)$ is an edge from the subtree of $v$ to a node $z$ on the tree path from $w$ to $v$.) If $(z, w) \in E$ then $(v, w)$ is reducible. Otherwise, if $(z, w) \notin E$ then we obtain:

1. The edge $(v, w)$ is reducible in $G$ if and only if the edge $(z, w)$ is reducible in the digraph $G_{1}=G-(v, w)+(z, w)$.

2. $G^{*}=G_{1}^{*}$.

3. Let $e=(a, b)$ be an edge in $G$ with $(v, w) \neq e \neq(z, w)$, $e \in B$, and there is no path $v \stackrel{*}{T} a$. Then $e$ is reducible in $G$ if and only if $e$ is reducible $G_{1}$.

Proof. Clearly, if $(z, w) \in E$ then the path $v \stackrel{*}{T} x \rightarrow z \rightarrow w$ is a reducing path relating to $(v, w)$, see figure 5 . On the other hand, let now $(v, z)$ not be an edge in $G$.

1. First we suppose $(v, w)$ is reducible in $G$. Then there exists a reducing path $P_{1}$ from $v$ to $w$ in $G$. $P_{1}$ is also a path in $G_{1}$. Now it can be enlarged through $z \stackrel{+}{T} v$ getting a path $P_{2}=z \stackrel{+}{T} w$. By shrinking cycles in $P_{2}$ we find a reducing path for $(z, w)$ in $G$. Conversely, suppose $P_{1}$ is a reducing path for $(z, w)$ in $G_{1}$. By our presumption there is a path $v \underset{T}{\rightarrow} x \rightarrow z$ in 


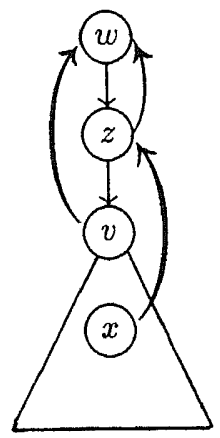

Figure 5

$G$. Joining this path with $P_{1}$ we obtain a $P_{2}=v \stackrel{+}{T} x \rightarrow z \stackrel{t}{\longrightarrow} w$ from $v$ to $w$ in $G$. Therefore, there also exists a simple path in $P_{3}$ of length at least two from $v$ to $w$ in $G$.

2. All nodes stay reachable in $G_{1}$ by replacing the edge $(v, w)$ through $v \stackrel{+}{T} x \rightarrow z \rightarrow w$. Otherwise we use the path $z \frac{t}{T} v \rightarrow w$ for the edge $(z, w)$ in $G$.

3. Let's first have the assumption: The edge $e=(a, b)$ is reducible in $G$ and the reducing path $P_{1}$ contains the edge $(v, w)$. The case $(v, w) \notin P_{1}$ is trivial. In order to observe a path $P_{2}$ connecting $a$ with $b$ in $G_{1}$ we apply replacement of edge $(v, w)$ by $v \frac{t}{T} x \rightarrow z \rightarrow w$. By our presumption this new path $P_{2}$ cannot embody the edge $(a, b)$. Hence shrinking cycles in $P_{2}$ gives a reducing path $P_{3}$ for $(a, b)$ in $G_{1}$. Inversely, suppose $(a, b)$ has a reducing path $P_{1}$ in $G_{1}$ holding within itself the edge $(z, w)$. The replacement of $(z, w)$ by $z \frac{+}{T} v \rightarrow w$ obtains a path $P_{2}$ from $a$ to $b$ in $G$. The lemma follows by same argumentation as above.

Now lemma 4 allows us the following method to test the reducibility of a backward edge $(v, w)$. By induction about the height of $v$ we assume that no backward edge $(x, y), x \neq v$, emanating from the subtree of $v$ is reducible. Then we calculate the node $z=z(v)$ defined by

(*) $\quad z=\min \{y \in V \mid \exists x \in V$ with $v \stackrel{+}{T} x$ and $(x, y) \in E\}$.

Note, since $G$ is strongly connected it is always $z \leq v$. If $z=v$ then $(v, w)$ is the only edge leaving the subtree of $v$ and $G-(v, w)$ would 
not be longer a strongly connected digraph. (Remember the observation about lowpoint $(v)$.) For that reason the edge $(v, w)$ is not reducible. Otherwise we delete and/or replace the edge $(v, w)$ by lemma 4 . This completes the induction step.

After this preparations we have all the results needed to build an efficient reducing algorithm for no-tree arcs, the algorithm A.

algorithm A

input: A strongly connected digraph $G(V, E)$; the partition of the edges $T, B, C, F$; the DFS-tree of $G$.

output: A subgraph $G^{\prime}=\left(V, E^{\prime}\right)$ of $G$, which does not contain a reducible no-tree arc.

(0) for $\forall e \in E$ do standfor $(e) \leftarrow e$ od;

for $\forall v \in V$ do backpoint $(v) \leftarrow v$ od;

(1) for $\forall e \in F$ dodelete $e$ from $G$ od;

(2) for $\forall e=(v, w) \in B$ do backpoint $(v) \leftarrow \min ($ backpoint $(v), w)$ od;

for $\forall e=(v, w \in B$ with $w \neq$ backpoint $(v)$ do delete $(v, w)$ from $G$ od;

(3) for $\forall e=(v, z) \in C$ do let $w$ be the nearest common ancestor of $v$ and $z$ if $w<$ backpoint $(v)$

then

add $(v, w)$ to $G$;

delete $(v$, backpoint $(v))$ from $G$;

standfor $(v, w) \leftarrow(v, z)$;

$\mathrm{fi}$ backpoint $(v) \leftarrow w$;

delete $(v, z)$ from $G$ od;

(4) $R$-test ("root of DFS-tree");

(5) replace all edges $e$ in $G$ by their origin standfor $(e)$

with

(6) $\quad R-\operatorname{test}(v: V)$

for $\forall w$ with $(v, w) \in T$ do $R-\operatorname{test}(w)$ od if $v$ is not a leaf 


\section{then}

$$
\begin{aligned}
& z \leftarrow \min (\{\text { backpoint }(w) \mid(v, w) \in T\}) ; \\
& \text { if } v \neq z \quad(* z<v *) \\
& \text { then } \\
& \quad \text { if backpoint }(v)<\text { backpoint }(z) \\
& \quad \text { then }
\end{aligned}
$$

\section{then}

$$
\begin{aligned}
& \text { delete }(z, \text { backpoint }(z)) \text { from } G \text {; } \\
& \text { add }(z, \text { backpoint }(v)) \text { to } G ; \\
& \text { standfor }((z, \text { backpoint }(v))) \leftarrow \operatorname{standfor}((v, \text { backpoint }(v))) \text {; } \\
& \text { backpoint }(z) \leftarrow \text { backpoint }(v) ;
\end{aligned}
$$

fi;

delete $(v$, backpoint $(v))$ from $G$;

$\mathrm{fi}$

backpoint $(v) \leftarrow z$

$\mathrm{fi}$

algorithm A

This algorithm works correctly by the results in preceding discussion. Let $G^{\prime \prime}\left(V, E^{\prime \prime}\right)$ be the digraph given by the algorithm A before step 5 is executed. This digraph $G^{\prime \prime}$ is constructed via the sequence of digraphs

$$
G=G_{0}, G_{1}, \ldots, G_{s}=G^{\prime \prime},
$$

where $G_{i+1}$ arises from $G_{i}$ by deletion of a reducible edge $e_{i}$, or by replacement of an edge $e_{i}^{\prime}$ by another $e_{i}^{\prime \prime}$ using lemma 3 or 4 . Therefore, we have $G_{i+1}^{*}=G_{i}^{*}$ and by induction on $i$ the fact $G_{o}^{*}=G_{s}^{*}$. If we replace $e_{i}^{\prime}$ by $e_{i}^{\prime \prime}$ then we keep the origin $e_{i}^{\prime}$ of $e_{i}^{\prime \prime}$ in the variable standfor, with standfor $\left(e_{i}^{\prime \prime}\right)=e_{i}^{\prime}$. With a replacement we do not change the reducibility of any edge $e, e \notin T$ and $e_{i}^{\prime} \neq e \neq e_{i}^{\prime \prime}$, see Lemma 3.3 or Lemma 4.3. In Lemma 4.3 the proof is only given for the case if $e=(a, b)$ is not an edge emanating from the subtree of $v$. But we see by induction on the height $(v)$ : If $e$ is emanating from the subtree of $v$ then $e$ is not reducible. Note, that the node $z=z(v)$ computed in $R$-test satisfies the definition in expression $(*)$. This completes our above proposition, since an edge which is not reducible in a digraph $G$ is also not reducible in any subgraph of $G$. So we obtain first that every remaining no-tree $\operatorname{arc} e$ in $G^{\prime \prime}$ is not reducible in $G^{\prime \prime}$. Secondly we have by induction on $i$ with starting node $i=s$ : If a no-tree arc $e$ is not reducible in $G^{\prime \prime}$ then its origin in $G$ is not reducible in $G$ too. Therefore, we can summarize our analysis in the following Theorem. 
Theorem 1. Algorithm A computes a strongly connected subgraph $G^{\prime}$ of a digraph $G$ where $G^{\prime}$ does not contain any reducible no-tree arc relating to a given DFS-tree. The algorithm $A$ has a linear time and storage complexity $O(|V|+|E|)$.

Proof. The correctness of Theorem 1 is clear by the preceding discussion. So we only have to show the complexity bound. First of all we use depthfirst search to get the input data structures of the algorithm in particular the DFS-tree and the partition of the edges into $T, B, F, C$. It is wellknown that this can be done with linear time and storage complexity. Now we come to algorithm A. It is trivial to see that step $0,1,2$ and 5 can be implemented in time $O(1)$ per considered edge. This is also true for the step 3 without calculation of the nearest ancestor $w$ for the node $v$ and $z$. Using a data structure by HAREL, TARJAN we can compute $w$ in time $O(1)$ and storage $O(n)$. Now, for any fixed vertex $v$ the procedure $\mathrm{R}$-test takes time linear in the number of edges emanating from $v$. So the total costs of R-test are linear in the number of edges referring to the calling time of R-test. Because we have linear complexity in each of our 6 steps we reach a linear time and storage complexity for the whole algorithm A too.

\section{The Reducibility of Tree Arcs}

Throughout this section we suppose that $G^{\prime}=\left(V, E^{\prime}\right)$ is the resulting ;ubgraph of algorithm A. Therefore, all no-tree arcs are not reducible. But for some of the tree arcs it is easy to see that they are not reducible, ;oo. Note, if an edge $(v, w)$ is reducible then there are at least two edges In $G$ emanating from $v$ and at least two edges entering $w$. We call tree arcs with this property critical. By this notation we only have to test critical edges for its reducibility. But this can be done in time $O(n)$, since the graph $G^{\prime}$ has a very special structure. In our discussion we use again the DFS-tree of the original digraph $G$. Note, that this tree is still a valid DFS-tree for $G^{\prime}$.

Let now $(v, w)$ be a critical edge. If $(v, w)$ is the only critical edge in $G^{\prime}$ then there is a simple way to test the reducibility of $(v, w)$. Namely, delete $(v, w)$ from $G^{\prime}$ and use depth-first search - or any other graph exploration algorithm - with starting node $v$. By the definition of reducibility the edge $(v, w)$ is reducible iff $w$ is reachable from $v$ in $G^{\prime \prime}=G^{\prime}-(v, w)$. This takes time $O\left(|V|+\left|E^{\prime}\right|\right)=O(n)$. Now, we want reach this time bound to test all critical edges. So we must be a little bit more clever. 
Let us remember our definition of a strongly connected digraph. The statement " $G^{\prime}\left(V, E^{\prime}\right)$ is strongly connected" stands for

$$
v \stackrel{*}{\underset{E^{\prime}}{\longrightarrow}} w \text { and } w \underset{E^{\prime}}{\stackrel{*}{\longrightarrow}} v \quad \forall v, w \in E .
$$

For this we infer that the digraph $G^{\prime}$ contains for every vertex $z$ a spanning tree $T_{1}$ with root $z=z\left(T_{1}\right)$ such that

$$
z \underset{T_{1}}{\stackrel{*}{\longrightarrow}} w \quad \forall w \in V
$$

and further that $G^{\prime}$ contains for every vertex $z^{\prime}$ a spanning sink tree $T_{2}$ with root $z^{\prime}$ such that

$$
w \underset{T_{2}}{\stackrel{*}{\longrightarrow}} z^{\prime} \quad \forall w \in V .
$$

This shows us the way to test all critical edges combined in one step. First we take away all critical edges from $G^{\prime}$. In general this new graph $G^{\prime \prime}$ is not strongly connected. Especially $G^{\prime \prime}$ does not contain a spanning tree with root $r=1$, the smallest node. So we have to add again some critical edges to get a spanning tree with root $r$. It is easy to see that the edges added in this step are not reducible in $G^{\prime}$. The second step is the analogous procedure for a spanning sink tree with root $r$. For all critical edges which are not added again we show that they are reducible in $G^{\prime}$.

By definition if the critical edge $e=(v, w)$ is reducible then ther exists a reducing path $P$ in $G^{\prime}$. Since $G^{\prime}$ does not contain any forward edge the reducing path from $v$ to $w$ must use a cross edge $e^{\prime}=(x, y)$ to enter the subgraph of $w$. By the definition of cross edges we have $w \leq y<x$. (Remember we suppose $d f s n u m(v)=v$.) For that reason we find the relevant observation for our first step: The set of all vertices greater than $w$ is a node separator for every path from $v$ to $w$ in $G^{\prime}-(v, w)$. Now we start a graph exploration algorithm to find the reachable node from the root $r$. If all vertices in $G^{\prime \prime}$ are reached then $G^{\prime \prime}$ has a spanning tree with root $r$ and we have finished. Otherwise, let $A$ be the set of vertices such that there is a critical edge $(y, x)$ for every vertex $x$ in $A$ with $y$ is reachable from $r$ but $x$ is not reachable from $r$ by edges in $G^{\prime \prime}$. Let now $b$ be the largest node in $A$ and $(a, b)$ the critical edge entering $b$. By the definition of $b$ we see: $b$ is not reachable from $r$ using any vertex greater than $b$. But the set of this vertices is a node separator for any path from $a$ to $b$ in $G^{\prime}$ - and in $G^{\prime \prime}$ too - and for that reason the edge $(a, b)$ cannot be reducible in $G^{\prime}$. To examine the remaining critical edges 
we make $(a, b)$ as not reducible, mark $b$ as reached and start again the graph exploration algorithm in the node $b$. If the algorithm stops again before all vertices are reached then we find a further critical edge $(c, d)$ which is not reducible in $G^{\prime \prime}+(a, b)$ by the same argumentation as above. Hence we find a set of no reducible critical edges

$$
B=\{(a, b),(c, d), \ldots\}
$$

by induction on the number of stops of the exploring algorithm. Here the reducibility refers to $G^{\prime}$ such that it holds:

i) The digraph $G^{\prime \prime \prime}\left(V, E^{\prime \prime \prime}\right)=G^{\prime \prime}\left(V, E^{\prime \prime}\right)+B$ is a subgraph of $G^{\prime}$ which does not contain any reducible edges referring to $G^{\prime}$.

Further by the algorithmic construction we obtain that:

ii) $G^{\prime \prime \prime}$ contains a spanning subgraph $T_{1}$ with root $r$ and this implies

$$
r \underset{T_{1}}{\stackrel{*}{\longrightarrow}} v \quad \forall v \in V .
$$

So we have finished the first part of our discussion. Let's now come to point two in which we enlarge the digraph $G^{\prime \prime \prime}$ by critical edges to guarantee a spanning sink tree in the resulting digraph $G^{(i v)}$. For the Sllowing argumentation in theorem 2 it is sufficient to show that new dges are reducible in $G^{(i v)}$ but not in $G^{\prime}$ on the contrary to add edges I the preceding case. This allows us a simplier method to guarantee .he existence of a spanning sink tree $T_{2}$ in $G^{(i v)}$. To compute a sink tree we traverse the edges in $G^{\prime \prime \prime}$ in opposite direction using depth-first search - or any other graph exploration algorithm - with starting node $r$. (Note, to keep up our usual notation we understand reachable in opposite direction in this context. Therefore, $v$ is reachable from $r$ means here $v \underset{G^{\prime \prime \prime}}{\stackrel{*}{\longrightarrow}} r$.) As in part one it is possible again that the algorithm stops before all nodes are reached. Then there is at least one node $v$ in the set $D$,

$$
D=\{x \in V \mid \exists(x, y) \in E,(x, y) \text { is critical and } y \text { is reached }\} .
$$

Now select one critical edge $(v, w)$, mark $(v, w)$ be not reducible, add it to a set $H$, mark $v$ be reached, and start again the algorithm in $v$. We 
repeat this procedure until all vertices are reached. Then the graph $G^{(i v)}$ is given by $G^{(i v)}=G^{\prime \prime \prime}+H$. By the construction we know about $G^{(i v) \text { : }}$

iii) $G^{(i v)}$ contains a spanning sink tree $T_{2}$, this implies

$$
v \underset{T_{2}}{\stackrel{*}{\longrightarrow}} r \quad \forall v \in V
$$

iv) Every edge $(x, y)$ in $H$ is not reducible in $G^{(i v)}$, because $(x, y)$ is the only edge in $G^{(i v)}$ which leaves the subtree of $x$ related to $T_{2}$. This completes our analysis. We sum up the results in theorem 2 .

Theorem 2. The subgraph $G^{(i v)}$ defined in the above discussion is a minimal transitive reduction of $G$.

Proof. First of all, we must show that all edges in $G^{(i v)}$ are not reducible in $G^{(i v)}$ and second of all that every critical edge not in $G^{(i v)}$ have a reducing path in $G^{(i v)}$. The first part is given by the construction of $G^{(i v)}$. All edges in $G^{\prime \prime}$ are not reducible in $G^{\prime}$ by definition and, therefore, they are also not reducible in the subgraph $G^{(i v)}$ of $G^{\prime}$. This is also true for the edges adding to $G^{\prime \prime}$ to obtain $G^{\prime \prime \prime}$ by expression i). Then expression iv) shows that no edge adding to $G^{\prime \prime \prime}$ getting $G^{(i v)}$ is reducible in $G^{(i v)}$. This completes the first part.

Let now $(v, w)$ be a critical edge not in $G^{(i v)}$. Then we know by th construction of $G^{\prime \prime \prime}$ there is a path $P_{1}=r \underset{T_{1}}{*} w$. By iii) the digraph $G^{(i v)}$ contains in addition a spanning sink tree $T_{2}$ too, hence it exists a path $P_{2}=v \underset{T_{2}}{\stackrel{*}{\longrightarrow}} r$ in $G^{(i v)}$. Concatenation gives a path $P_{3}=v \underset{T_{2}}{\stackrel{*}{\longrightarrow}} \underset{T_{1}}{\stackrel{*}{\longrightarrow}} w$ which obtains the reducing path for $(v, w)$ in $G^{(i v)}$ by shrinking cycles.

The last point to show is that $G^{(i v)}$ is strongly connected. But this is easy because we have

$$
v \underset{T_{2}}{\stackrel{*}{\longrightarrow}} r \underset{T_{1}}{\stackrel{*}{\longrightarrow}} w \underset{T_{2}}{\stackrel{*}{\longrightarrow}} r \stackrel{*}{T_{1}} v \quad \forall v, w \in V
$$

Now we come to the implementation of the graph exploration algorithm referring to the construction of $G^{\prime \prime \prime}$ respectively $G^{(i v)}$. To make this for $G^{\prime \prime \prime}$ we must change our version of depth-first search. We must distinguish between critical and no critical edges. The no critical edges 
algorithm B

procedure explore $(v: V)$;

begin

add $v$ to $R E A C H$;

if $v \in A$ then delete $v$ from $A$ fi;

for $\forall w$ with $(v, w) \in E$ do

od;

if $w \notin R E A C H$ then explore $(w) \mathbf{f i}$

for $\forall w$ with $(v, w) \in E_{c}$ do

if $w \notin(R E A C H \cup A)$ then add $w$ to $A \mathbf{f i}$ od

end

Herewith the procedure explore is applied in the following way:

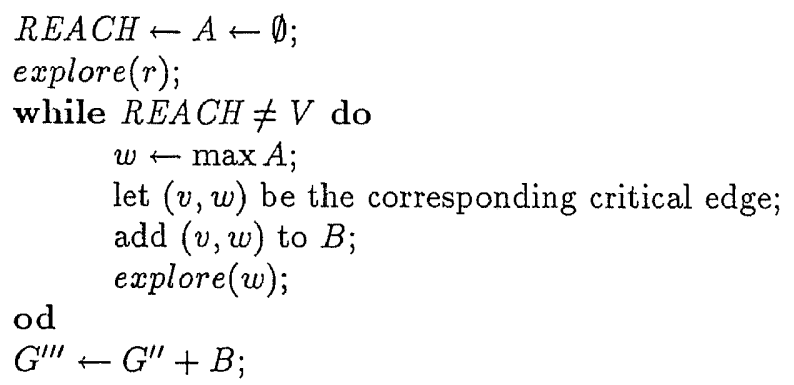

are kept in the normal edge set $E$ and the critical edges in $E_{c}$. In our discussion we use a set

$$
A=\left\{y \in(V-R E A C H) \mid \exists(x, y) \in E_{c} \text { with } x \in R E A C H\right\} .
$$

During the algorithm we hold this set $A$ in an array $A$. So we get the algorithm B with input graph $G^{\prime \prime}$. Let's have a look on it.

It is easy to see that this algorithm can be implemented straight forward in time $O(n)$ except the line

$$
w \leftarrow \max A .
$$


Because our insertions in $A$ are not monotonous this is the classical case of a heap under the operations insert and delete maximum for a bounded universe. We do not know any amortised solution for $n$ such operations in time $O(n)$ in contrary to our aim. But we can make our insertions monotonous. The adjective "monotonous" means that the operation in$\operatorname{sert}(x)$ is only used for arguments $x$ with

$$
x \leq \max A
$$

Our method is based on the following observation.

Let us consider the path $P=r \stackrel{*}{T} n$ on the DFS-tree $T$ in $G$ from the root $r$ to the largest node $n$. Let $(x, y)$ be an edge on this path $P$. We then see that $(x, y)$ cannot be reducible since a reducing path $P_{1}$ for $(x, y)$ has to use a cross edge to enter the subtree of $y$ in $G$. But there are no cross edges entering the subtree of $y$ by the definition of $n$. (Remember $n=d f \operatorname{snum}(n))$.

Let now $v^{\prime}$ be the maximum of $V-R E A C H$. First we see that $v^{\prime}$ is a node in the subtree of $v$ because $A$ is a subset of $V-R E A C H$. Therefore, it exists a path $P_{1}=v \underset{T}{\longrightarrow} v^{\prime}$. If now $(x, y)$ is an edge on the path $v \underset{T}{*} v^{\prime}$ then the above observation is also true for $(x, y)$. Note, if we suppose it is a reducing path for $(x, y)$ in $G^{\prime \prime}$ then either $v$ is not the maximum o $A$ or $v^{\prime}$ is not the maximum of $V-R E A C H$. Hence, every edge on thi: path $P_{1}$ is not reducible.

For that reason we can replace

$$
v \leftarrow \max A
$$

by
a)
$v^{\prime} \leftarrow \max (V-R E A C H) ;$
b)
let $P=v \underset{T}{\stackrel{*}{\longrightarrow}} v^{\prime}=v_{0}, \ldots, v_{s}$ with $v_{0}=r, v^{\prime}=v_{s}$.
c)
$v \leftarrow \max \left\{v_{i} \mid v_{i} \in A\right\}$

Then we find $v^{\prime}$ by sliding a pointer $p t$ cross the bitvector $R E A C H$. All elements to right of $p t$ are not in $R E A C H$, where $p t$ starts with $p t=n$. This implementation is possible since the insertions in $V-R E A C H$ are monotonous in natural way. So the computation of $v^{\prime}$ has amortised running time $O(n)$. To calculate $v$ we follow the tree path $P^{\prime}$ from $v^{\prime}$ to the root until the first node which is in $A$. Then the vertex $v$ is found. 
For all critical edges $e$ on $P^{\prime}$, we delete $e$ from $E_{c}$ and add $e$ to $E$ and $B$. This implies an amortised time of $O(n)$ for the step b) since all vertices on the path $P^{\prime}=v^{\prime} \underset{T}{\stackrel{*}{\longrightarrow}} v$ will be reached in the next call explore $(v)$. All arguments combined give a total running for explore of $O(n)$.

Now, the procedure explore is also useful to compute $G^{(i v)}$. First we turn around the direction of the edges in $G^{\prime \prime \prime}$ and we next replace the statement

$$
v \leftarrow \max A
$$

through

select any vertex $v$ from $D$,

where the name $D$ is used for $A$ and $H$ for $B$ getting a procedure explore'. Then explore computes $G^{(i v)}$ in time $O(n)$ since it is a simpler version of explore. This completes the examination of tree arcs. Therefore, we can summarize all the results together in Theorem 3 .

Theorem 3. A minimal transitive reduction of a given strongly connected digraph $G(V, E)$ is computable in linear time $O(|V|+|E|)$.

Proof. By algorithm $A$ we find in linear time $O(n+m)$ a subgraph $G^{\prime}$ with $G^{*}=\left(G^{\prime}\right)^{*}$ which has not any reducible no-tree arcs. Since $G^{\prime}$ contains at most $2 \cdot n$ edges the calculation of $G^{\prime \prime}$ costs $O(n)$, because the decision whether an edge is critical can be done in $O(1)$ per edge. The procedure explore takes time $O(n)$ to compute $G^{\prime \prime \prime}$ from $G^{\prime \prime}$ and in the same way explore $e^{\prime}$ results in a minimal transitive reduction $G^{(i v)}$.

\section{Conclusions and Open Problems}

This paper has presented an algorithm for finding a minimal transitive reduction of a given strongly connected digraph $G$. The algorithm is based on depth-first search partly as an algorithm and partly as induced data structure. Further, the algorithm uses some graph transformations technical similar to transformations in TARJAN, 1974. The running time is given by $O(n+m)$, therefore, the algorithm is optimal to within a constant factor. Although the algorithm consists of several algorithmic parts, it should not be very difficult to program.

We think that the following questions are of some interest in view of the minimal transitive reduction. Is it possible to decide whether digraph $G$ is Bellman-Ford-Orderable on its minimal transitive reduction? Is 
there a relation between the transitive reduction of a digraph $G$ and its dominators?

\section{References}

[1] A.V. Aho, M.R. Garey and J.D. Ullman: The Transitive Reduction of a Directed Graph, SIAM J. Computing, 1 (1972), 131137.

[2] V.L.Arlazarov, E.A. Dinic, M.A. Kronod a. J.A. Faradzev; On economical construction of the transitive closure of an oriented graph, Dokl. Akad. Nauk SSSR 11, (1970), 1209-1210.

[3] M. O'hEigeartaigh, J.K. Lenstra, A.H.G. Rinnooy Kan: Combinatorial Optimization, John Wiley and Sons, New York, Annotated Bibliographies, 1985.

[4] S. Even: Graph Algorithms, Computer Science Press, Potomac, MD, 1979.

[5] M.J. Fischer AND A.R. Meyer: Boolean matrix multiplication and transitive closure, Conference Record, Twelfth Annual Symposium on Switching and Automata Theory, East Lansing, Mich., 1971, 129-131.

[6] A. Goralcikova, V. Koubek: A Reduct and Closure algorithm for Graphs, Mathematical Foundations of Computer Science 79, Sprin ger Lecture Notes in Computer Science 74, 301-307.

[7] D. Harel and R.E. TARJaN: Fast Algorithms for Finding Nearest Common Ancestors, SIAM J. Computing 13 (1984), 339-355.

[8] H.T. Hsu: An Algorithm for Finding a Minimal Equivalent Graph of a Digraph, J. ACM 22 (1975), 11-16.

[9] K. Mehlhorn: Data Structures and Algorithms, Vol 2: Graph Algorithms and NP-Completeness, Springer, EATCS Monographs in Computer Science, 1984.

[10] K. Mehlhorn and B. H. Schmidt: On BF-Orderable Graphs Discrete Applied Mathematics 15(1986), 315-327

[11] D.M. Moyles AND G.L. Thompson: Finding a minimum equivalent graph of a digraph, J. ACM 16 (1969), 455-460.

[12] P. Purdom: A transitive closure algorithm, BIT 10 (1979), 76-94.

[13] R. HADDAD, A. SchäfFER: Recognizing Bellman-Ford-Orderable Graphs, Computer Science Department Stanford University, Standford, California 94305-2140. 
[14] S. SAHNI: Computationally Related Problems, SIAM J. Computing 3 (1974), 262-279.

[15] C.P. SchnorR: An Algorithm for Transitive Closure with Linear Expected Time, SIAM J. Computing 7 (1978), 124-133.

[16] K. Simon: An Improved Algorithm for Transitive Closure on Acyclic Digraphs, Theoretical Computer Science 58 (1988), 325-346.

[17] K. Simon: On Minimum Flow and Transitive Reduction, Proceedings ICALP'88, Tampere, Lecture Notes in Computer Science, Springer-Verlag, 317, 535-560.

[18] R.E. TARJAN: Depth first search and linear graph algorithms, SIAM J. Computing 1 (1972), 146-160.

[19] R.E. TARJAN: Finding Dominators In Directed Graphs, SIAM J. Computing 3 (1974), 62-89.

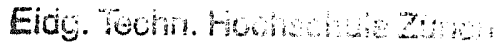

Informaikbibl ohet

ETH-Zentrum

CH-8092 Zürich 